\title{
Magnetic resonance imaging findings in children with spasmus nutans
}

Meredith Bowen, BA, ${ }^{\mathrm{a}}$ Jason Peragallo, MD, ${ }^{\mathrm{b}, \mathrm{c}}$ Stephen F. Kralik, MD, ${ }^{\mathrm{d}}$ Andrea Poretti, MD, Thierry A.G.M. Huisman, MD, ${ }^{\mathrm{e}}$ Bruno P. Soares, MD,

Author affiliations: ${ }^{a}$ Emory University School of Medicine, Atlanta, Georgia $;{ }^{b}$ Department of Ophthalmology, Emory University School of Medicine, Atlanta, Georgia; ${ }^{c}$ Department of Pediatrics, Emory University, Atlanta, Georgia; ${ }^{d}$ Section of Neuroradiology, Department of Radiology and Imaging Sciences, Indiana University, Indianapolis, Indiana; ${ }^{e}$ Section of Pediatric Neuroradiology, Division of Pediatric Radiology, Russell H. Morgan Department of Radiology and Radiological Science, The Johns Hopkins University School of Medicine, Baltimore, Maryland

Submitted September 13, 2016.

Revision accepted November 23, 2016.

Correspondence: Bruno P. Soares, MD, The Johns Hopkins Hospital, 1800 Orleans Street, Zayed Tower, Rm 4174, Baltimore, MD 21287 (email: bruno.soares@jhmi.edu).

\section{Word count: 1,667}

Abstract only: 253

This is the author's manuscript of the article published in final edited form as:

Bowen, M., Peragallo, J. H., Kralik, S. F., Poretti, A., Huisman, T. A. G. M., \& Soares, B. P. (n.d.). Magnetic resonance imaging findings in children with spasmus nutans. Journal of American Association for Pediatric Ophthalmology and Strabismus. https://doi.org/10.1016/j.jaapos.2017.03.001 


\begin{abstract}
Background

Spasmus nutans $(\mathrm{SN})$ is a rare pediatric ophthalmologic syndrome characterized by nystagmus, head bobbing, and abnormal head positioning. Historically, SN has been associated with underlying optic pathway gliomas (OPG); however, evidence of this association is based primarily on a small number of isolated case reports. Prior retrospective analyses have found the rate of OPG to be $<2 \%$, but these studies only intermittently used neuroimaging with computed tomography, which has limited sensitivity for detection of small lesions in the optic pathway.

The purpose of this study was to investigate the association of SN with intracranial abnormalities, particularly OPG, using magnetic resonance imaging of the brain and orbits.
\end{abstract}

\title{
Methods
}

Neuroradiology databases at three institutions spanning January 2010 to May 2016 were queried for examinations ordered for evaluation of SN; MRI examinations of the brain and/or orbits were included and evaluated for OPG and other structural abnormalities. Medical records were reviewed to confirm a diagnosis of $\mathrm{SN}$, presence of other underlying neurological disease, or preexisting diagnoses.

\section{Results}

A total of 40 patients with eligible MRI examinations were identified. None had optic nerve pathway gliomas. Two children had optic nerve hypoplasia; no other patients had optic pathway abnormalities. None had intracranial or orbital masses. MRI examinations were normal in 25 patients.

\section{Conclusions}

This series represents the largest collection of MRI examinations for SN in the literature to date 
and shows no association between OPG and SN. In children presenting with SN but no other findings concerning for OPG or neurological abnormalities, neuroimaging may not be required. 
Spasmus nutans $(\mathrm{SN})$ is a rare pediatric ophthalmologic syndrome that was first described in the English-language literature in 1901 is defined by the triad of nystagmus, head-nodding or titubation, and anomalous head position, such as torticollis, with onset in the first year of life. ${ }^{1}$ The nystagmus is typically intermittent, of small amplitude and high frequency, variably disconjugate or disjunctive, greater in the abducting eye, and may have a vertical component. Head nodding is irregular and may have horizontal and vertical components. Strabismus and amblyopia may coexist, and SN may also be associated with or a mimicker of underlying retinal pathology. ${ }^{2,3}$ Historically, however, SN has been associated with underlying optic pathway gliomas, prompting contrast-enhanced neuroimaging to become a common component of its diagnostic work up. ${ }^{4-7}$ However, the evidence of this is association is based primarily on a small number of isolated case reports dating back to the 1970s. ${ }^{6,8-12}$

Several prior retrospective studies of children with SN-like nystagmus have found no increased incidence of optic pathway gliomas, with rates ranging from $<2 \%$ to $9 \%$. ${ }^{13-15}$ However, most of these studies suffered from small series sizes and used only lower-resolution computed tomography (CT). We performed a systematic analysis of a cohort of 40 children diagnosed with SN and subsequently undergoing magnetic resonance imaging (MRI) with the goal of evaluating the prevalence of intracranial abnormalities, particularly optic pathway gliomas.

\section{Subjects and Methods}

This study was approved by the local institutional review boards of Emory University, Indiana University, and Johns Hopkins University. Neuroradiology databases of Children's Hospital of Atlanta, Riley Hospital for Children, and Johns Hopkins Children's Center spanning the period from January 2010 to May 2016 were queried for examinations ordered for evaluation of SN using searches for "spasmus nutans" as well as associated misspellings, including "spasms" and 
"mutans."

All patients with MRI ordered for SN were included. Clinical diagnosis of SN was made prior to imaging by pediatric ophthalmologists or neurologists. Electronic medical records, when available, were examined for concomitant underlying neurological disease; for known, preexisting systemic disorders, including genetic syndromes and chromosomal abnormalities; and for symptoms consistent with SN (nystagmus, head nodding, and anomalous head position). Additionally, available ophthalmology notes were examined for signs of underlying intraorbital or optic pathway abnormality, such as reduced visual acuity and strabismus. Age at symptom onset was also collected when available.

Brain and orbit MRI studies were performed according to institutional protocols using 1.5 or 3.0 T clinical MR scanners. At minimum, imaging protocols included standard brain MRI sequences such as sagittal T1-weighted spin echo (SE) or 3D T1 gradient echo (GRE), axial T2weighted fast spin echo and T2 fluid-attenuated inversion recovery and, if contrast was administered, postcontrast coronal and axial T1 SE or sagittal 3D T1 GRE. The majority of patients had dedicated orbital MRI sequences such as thin-slice coronal T2 turbo-spin echo and fat-suppressed contrast-enhanced T1 SE. MRI examinations were considered eligible for inclusion when the optic apparatus could be properly assessed. All available MRI studies were evaluated for optic pathway gliomas and other structural brain abnormalities by a subspecialty certified neuroradiologist with 6 years of experience in pediatric neuroimaging (BPS).

\section{Results}

A total of 40 patients (21 males) with MRI of the brain and/or orbits ordered for evaluation of SN were included: 31 at Children's Hospital of Atlanta, 4 at Riley Hospital for Children, and 5 at Johns Hopkins Children's Center. The median age at time of imaging was 16.3 months 
(interquartile range, 17.4 months).

Of 13 children with complete ophthalmology notes available, 5 had clinically isolated SN. Four children had decreased visual acuity, 4 had esotropia, 1 child had astigmatism, 1 had retinal dystrophy, and 1 had familial exudative vitreoretinopathy. No patient had relative afferent pupillary defect or optic disk pallor.

Of the 31 patients for whom medical records at the time of SN diagnosis were available, 16 had no previously known underlying disease or developmental delay at the time of imaging; 15 did have significant, systemic, nonocular diagnoses, including trisomy 21 (7), cerebral palsy (2), developmental delay of unknown etiology (5) and a 1p36 chromosomal deletion syndrome (1).

On imaging review, 25 patients (63\%) had normal, age-appropriate MRI examinations. No patients had optic pathway gliomas. Two patients had bilateral optic nerve hypoplasia (Figure 1); no other patients had any optic pathway abnormalities. Intracranial abnormalities were found in 15 patients (Table 1$)$.

Of the 16 known developmentally normal, otherwise healthy children, 11 had normal MRI examinations. The abnormal findings included mild ventriculomegaly, periventricular nodular heterotopia, large subarachnoid spaces, and a complex pineal cyst. There was no significant difference between the frequency of abnormal MRI examinations in otherwise healthy children compared to children with known delays $(P=0.38)$.

\section{Discussion}

Spasmus nutans can be confused with other entities. The nystagmus of SN is asymmetric-even monocular—pendular, low amplitude, and high frequency. ${ }^{14,16}$ Symptoms most commonly arise between 6 and 36 months of age and typically resolve within several years without harm to 
visual acuity, although subclinical nystagmus can be noted as many as 7 years after diagnosis. ${ }^{17-}$ ${ }^{19}$ Antony and colleagues ${ }^{7}$ presented cases of children "misdiagnosed" with SN who were ultimately found to have intracranial tumors; one also had persistent, severe failure to thrive, another, low body weight-to-head circumference and optic disk pallor. ${ }^{7}$ Head nodding or head titubation may be seen in other diseases including rhombencephalosynapsis, Joubert syndrome, "bobble-head doll" syndrome, congenital nystagmus, shuddering attacks, and STXBP1 encephalopathy. ${ }^{20}$ Differentiation between head nodding or head titubation in SN and head movements in other disorders is usually possible based on the clinical characteristics of the head movements and additional neurological findings.

When optic pathway gliomas have been found with SN as an early symptom, it has usually been in the setting of other symptoms or signs considered red flags. Kiblinger and colleagues ${ }^{15}$ found 2 cases of optic gliomas in 20 imaged children; however, both of these children presented with $\mathrm{SN}$ at a later age than the average presentation, and neither child had the classic SN triad; both were noted to have optic disk pallor on their initial examination, and one had been previously diagnosed with neurofibromatosis. ${ }^{15}$ In addition, the spontaneous resolution of SN with age is well compatible with the lack of an underlying structural abnormality and might rather reflect either structural maturation of the brain or "full calibration" of eye movements, although spontaneous resolution of $\mathrm{SN}$ does not preclude the absence of an underlying lesion. ${ }^{11}$

This study of 40 children with a diagnosis of SN evaluated with MR imaging shows that there is no apparent association between optic pathway gliomas and SN. The absence of underlying gliomas is consistent with prior series of $\mathrm{SN}$ that have included neuroimagingalmost exclusively CT_-which reported a prevalence of optic pathway gliomas in zero of 14 
patients $^{14}$ and zero of 41 patients. ${ }^{13}$

Our data suggests that although some patients with SN may have underlying structural abnormalities found on imaging, the risk of optic gliomas in children without other neurological deficits or signs concerning for intracranial mass lesions is very low. Using the simplistic assumption that the next patient enrolled in our cohort would have an optic glioma-as was assumed in a prior study ${ }^{13}$ - would result in an incidence of one in 41 , or $2.4 \%$. Similarly, by the binomial exact test, the association between $\mathrm{SN}$ and optic pathway gliomas is low, with a $95 \%$ confidence interval for nonassociation of 0.91-1.00; larger studies are warranted for further, more detailed statistical analysis.

This study has several limitations, including those inherent to its retrospective design. One institution contributed the majority of patients (31). Not all patients underwent dedicated orbital MRI, and there was some heterogeneity in sequences used. However, all MRI examinations were considered of sufficient quality to properly assess the brain and optic apparatus. Not all patients had detailed ophthalmology notes available for review; thus the misdiagnosis of SN rather than another eye-movement disorder cannot be entirely ruled out, especially in children with underlying systemic disorders associated with nystagmus, including as trisomy $21 .^{21-24}$ Lastly, the clinical diagnosis of SN may have been variable, given its low prevalence.

This series further confirms that neuroimaging — often requiring sedation given the young age of the patients in question-is not always necessary as part of initial diagnostic work-up in children diagnosed with or being evaluated for spasmus nutans. Based on our findings, we believe a period of watchful waiting prior to imaging is reasonable in children with clinical diagnosis of spasmus nutans but no additional concerning findings on physical examination or 
history.

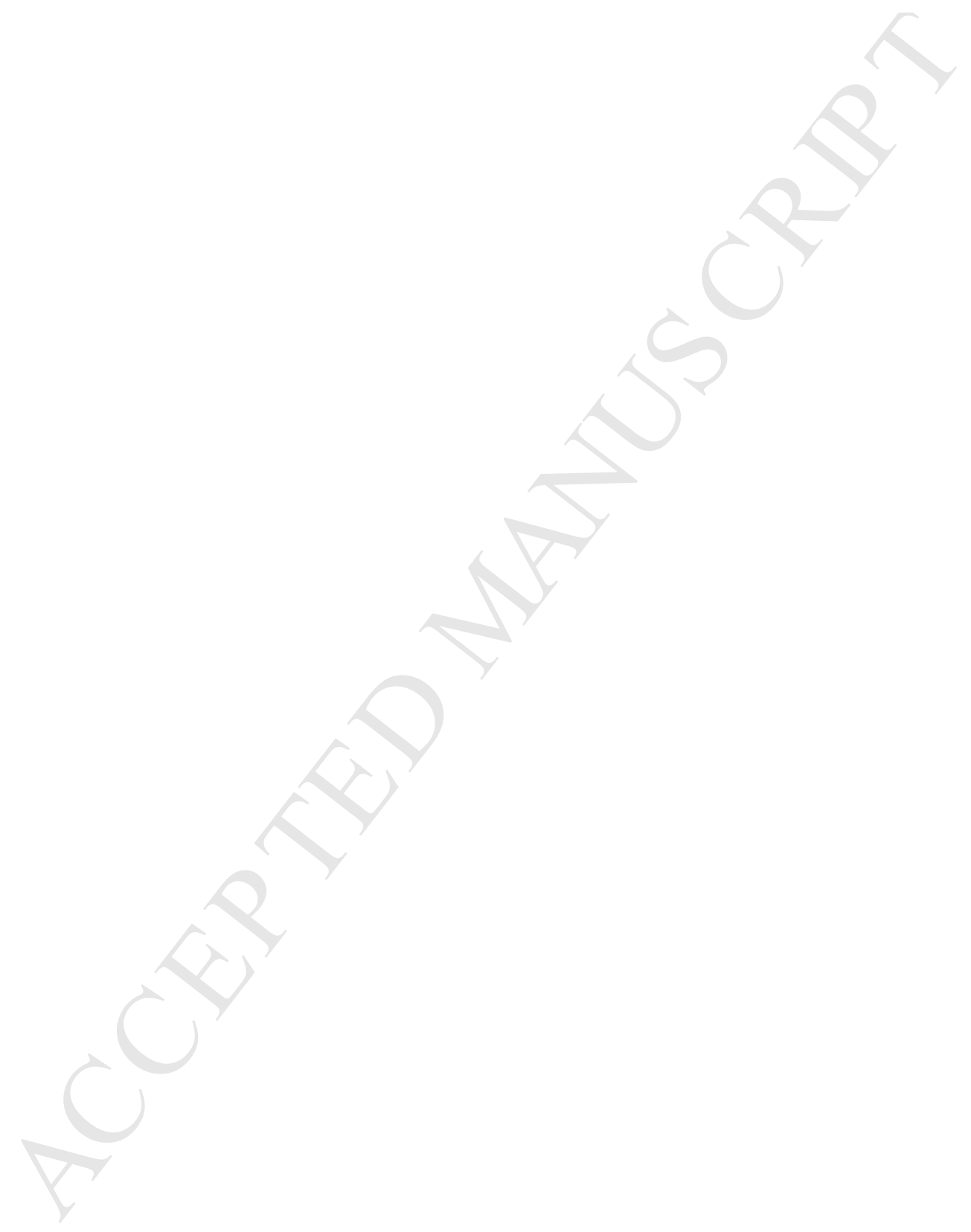




\section{References}

1. Thomson J. Note on the peculiar nystagmus of spasmus nutans in infants. Br Med J $1901 ; 1: 763$.

2. Gottlob I, Wizov SS, Reinecke RD. Quantitative eye and head movement recordings of retinal disease mimicking spasmus nutans. Am J Ophthalmol 1995;119:374-6.

3. Smith DE, Fitzgerald K, Stass-Isern M, Cibis GW. Electroretinography is necessary for spasmus nutans diagnosis. Pediatr Neurol 2000;23:33-6.

4. Shaw FS, Kriss A, Russel-Eggitt I, Taylor D, Harris C. Diagnosing children presenting with asymmetric pendular nystagmus. Dev Med Child Neurol 2001;43:622-7.

5. Skorin L, Jr., Briggs KS, Multack RF. Spasmus nutans: a pediatric enigma. J Am Optom Assoc 1986;57:893-4.

6. Kelly TW. Optic glioma presenting as spasmus nutans. Pediatrics 1970;45:295-6

7. Antony JH, Ouvrier RA, Wise G. Spasmus nutans: a mistaken identity. Arch Neurol $1980 ; 37: 373-5$

8. Albright AL, Sclabassi RJ, Slamovits TL, Bergman I. Spasmus nutans associated with optic gliomas in infants. J Pediatr 1984;105:778-80.

9. Lavery MA, O’Neill JF, Chu FC, Martyn LJ. Acquired nystagmus in early childhood: a presenting sign of intracranial tumor. Ophthalmology 1984;91:425-53.

10. Schulman JA, Shults WT, Jones JM Jr. Monocular vertical nystagmus as an initial sign of chiasmal glioma. Am J Ophthalmol 1979;87:87-90.

11. Brodsky MC, Keating GF. Chiasmal glioma in spasmus nutans: a cautionary note. J Neuroophthalmol 2014;34:274-5.

12. Koenig SB, Naidich TP, Zaparackas Z. Optic glioma masquerading as spasmus nutans. J 
Pediatr Ophthalmol Strabismus 1982;19:20-24.

13. Arnoldi KA, Tychsen L. Prevalence of intracranial lesions in children initially diagnosed with disconjugate nystagmus (spasmus nutans). J Pediatr Ophthalmol Strabismus 1995;32:296-301.

14. King RA, Nelson LB, Wagner RS. Spasmus nutans: a benign clinical entity? Arch Ophthalmol 1986;104:1501-4.

15. Kiblinger GD, Wallace BS, Hines M, Siatkowski RM. Spasmus nutans-like nystagmus is often associated with underlying ocular, intracranial, or systemic abnormalities. J Neuroophthalmol 2007;27:118-22.

16. Olitsky S, Hug D, Plummer L, et al. Disorders of eye movement and alignment. In: Kliegman RM, Stanton BF, St. Geme JW, Schor NF, eds. Nelson Textbook of Pediatrics. Philadelphia, PA: Elsevier Saunders; 2016.

17. Gottlob I, Reinecke RD. Eye and head movements in patients with achromatopsia. Graefes Arch Clin Exp Ophthalmol 1994;232:392-401.

18. Gottlob I, Wizov SS, Reinecke RD. Spasmus nutans: a long-term follow-up. Invest Ophthalmol Vis Sci 1995;36:2768-71.

19. Norton EW, Cogan DG. Spasmus nutans; a clinical study of twenty cases followed two years or more since onset. AMA Arch Ophthalmol 1954;52:442-6.

20. Poretti A, Christen HJ, Elton LE, et al. Horizontal head titubation in infants with Joubert syndrome: a new finding. Dev Med Child Neurol 2014;56:1016-20.

21. Akinci A, Oner O, Bozkurt OH, Guven A, Degerliyurt A, Munir K. Refractive errors and strabismus in children with Ddown syndrome: a controlled study. J Pediatr Ophthalmol Strabismus 2009;46:83-6. 
22. Creavin AL, Brown RD. Ophthalmic abnormalities in children with Down syndrome. J Pediatr Ophthalmol Strabismus 2009;46:76-82.

23. Doummar D, Roussat B, Beauvais P, Billette de Villemeur T, Richardet JM. Spasmus nutans: a series of 16 cases. Arch Pediatr 1998;5:264-8.

24. Dumitrescu AV, Moga DC, Longmuir SQ, Olson RJ, Drack AV. Prevalence and characteristics of abnormal head posture in children with Down syndrome: a 20-year retrospective, descriptive review. Ophthalmology 2011;118:1859-64. 


\section{Legends}

FIG 1. Optic nerve hypoplasia, found in 2 patients, was the most common intracranial abnormality in our study cohort and the only optic pathway abnormality. Its association with spasmus nutans has not been established. Axial (A) and coronal (B) T2-weighted magnetic resonance images show symmetrically diminished caliber of the optic nerves (arrows) with normal signal intensity, consistent with hypoplasia. Coronal (C) T2-weighted image of a different patient shows, for comparison, normal-sized optic nerves. 
Table 1. Intracranial abnormalities on magnetic resonance imaging ordered for children with suspected spasmus nutans

\begin{tabular}{|c|c|}
\hline MRI findings & Medical history, if known \\
\hline $\begin{array}{l}\text { Agenesis (partial) of posterior body and splenium of corpus } \\
\text { callosum, malrotated hippocampi }\end{array}$ & Trisomy 21, premature \\
\hline Brachycephaly, mild-to-moderate ventriculomegaly & Developmental delay \\
\hline Bulging at lateral aspect of bilateral globes & $\begin{array}{l}\text { Otherwise healthy; known history of familial } \\
\text { exudative vitreoretinopathy }\end{array}$ \\
\hline Complex pineal cyst without hydrocephalus & Otherwise healthy \\
\hline Corpus callosum thinning & Global developmental delay, premature \\
\hline $\begin{array}{l}\text { Corpus callosum thinning, mild ventriculomegaly, diminished } \\
\text { periventricular white matter }\end{array}$ & $\begin{array}{l}1 \text { p36 chromosomal deletion, resultant } \\
\text { seizure disorder }\end{array}$ \\
\hline Frontal lobe: large subarachnoid spaces & Otherwise healthy \\
\hline $\begin{array}{l}\text { Intraventricular hemorrhage/germinal matrix hemorrhage } \\
\text { (mild, chronic) }\end{array}$ & Unknown \\
\hline Optic nerve hypoplasia, bilateral & Global developmental delay \\
\hline Periventricular leukomalacia (mild) & Unknown \\
\hline Periventricular nodular heterotopia & Otherwise healthy \\
\hline Ventricles, prominent, and bilateral optic $\mathrm{r}$ & Unknown \\
\hline Ventriculomegaly (mild) & Otherwise healthy \\
\hline Ventriculomegaly with paucity of white matter without gliosis & Developmental delay \\
\hline
\end{tabular}




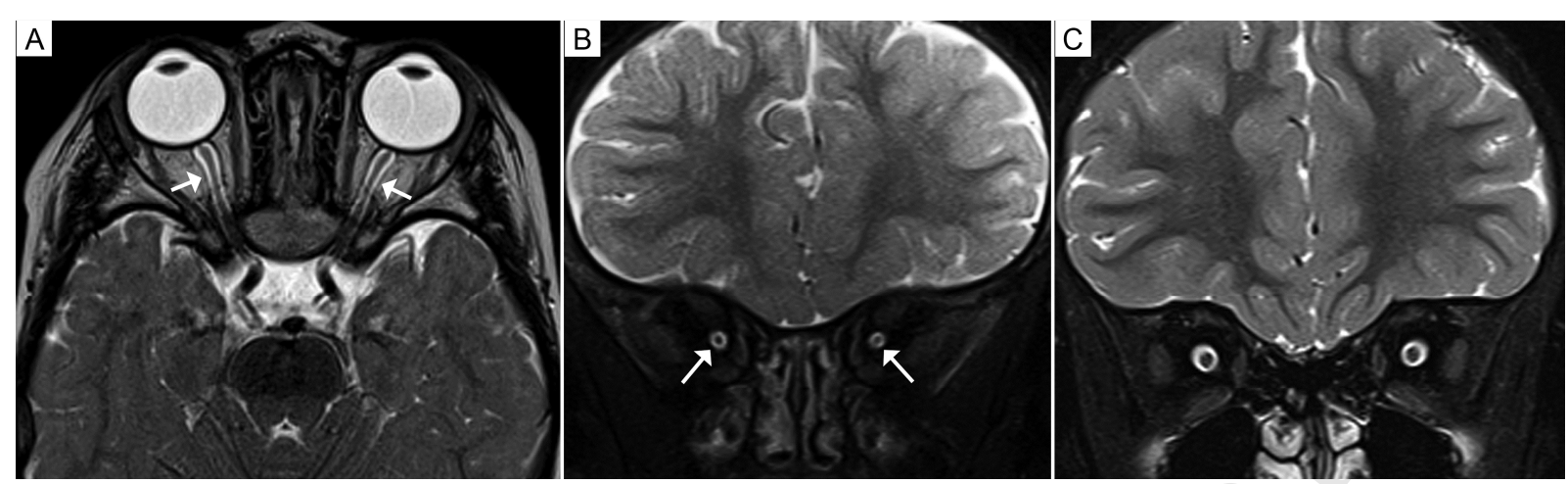

